\title{
Neoadjuvant chemotherapy with biosimilar trastuzumab in human epidermal growth factor receptor 2 overexpressed non-metastatic breast cancer: patterns of use and clinical outcomes in India
}

\author{
Anjana Joel ${ }^{1}$, Josh Thomas Georgy ${ }^{1}$, Divya Bala Thumaty ${ }^{1}$, Ajoy Oommen John ${ }^{1}$, Raju Titus Chacko ${ }^{1}$, Grace Rebekah ${ }^{2}$, \\ Elanthenral Sigamani ${ }^{3}$, Jagan Chandramohan ${ }^{3}$, Marie Therese Manipadam³ , Anish Jacob Cherian ${ }^{4}$, Deepak Thomas Abraham ${ }^{4}$, \\ Paul Mazhuvanchary Jacob ${ }^{4}$, Patricia Sebastian ${ }^{5}$, Selvamani Backianathan ${ }^{5}$ and Ashish Singh ${ }^{1}$
}

${ }^{1}$ Department of Medical Oncology, Christian Medical College, Vellore 632004, India
${ }^{2}$ Department of Biostatistics, Christian Medical College, Vellore 632004, India
${ }^{3}$ Department of Pathology, Christian Medical College, Vellore 632004, India
${ }^{4}$ Department of Endocrine Surgery, Christian Medical College, Vellore 632004, India
${ }^{5}$ Department of Radiation Therapy, Christian Medical College, Vellore 632004, India

\section{Abstract}

Background: Human epidermal growth factor receptor 2 (HER2)-positive breast cancer is associated with poor prognosis and access to anti-HER2 treatment is still a challenge in lower-middle income countries. The availability of the biosimilar trastuzumab has improved access by lowering the costs. We report the pattern of use of neoadjuvant \pm adjuvant trastuzumab and outcomes in patients with HER2-positive non-metastatic breast cancer treated with regimens incorporating shorter durations of therapy and the use of the biosimilar trastuzumab compared to the innovator.

Methods: We conducted a retrospective analysis of patients with non-metastatic HER2positive breast cancer treated with neoadjuvant \pm adjuvant trastuzumab (innovator ( $n$ $=34(33 \%))$ and biosimilar $(n=70(67 \%))$ manufactured by Biocon Biologics) with chemotherapy. Information regarding chemotherapy regimens, duration of trastuzumab use ( $\leq 12$ weeks and $>12$ weeks), pathological response (Miller Payne grade), disease free survival (DFS), overall survival (OS) and safety data were collected from electronic medical records.

Results: A total of 135 patients were analysed with a median age of 51 years (range: 23-82); of these, $57 \%$ were postmenopausal, $31.8 \%$ were hormone receptor positive and $62.9 \%$ had stage III disease. The overall pathological complete response ( $p-C R$ ) in both breast and axilla increased to $37.6 \%$ in patients treated with trastuzumab preoperatively as compared to $22.2 \%$ in patients who did not receive any trastuzumab. Patients receiving innovator trastuzumab and biosimilar trastuzumab showed a $\mathrm{p}-\mathrm{CR}$ of $28.5 \%$ and $41.7 \%$, respectively. At a median follow-up of 42 months (range: 3-114), there were 18 relapses and 11 deaths. The 3 -year DFS was $87.1 \%$ and OS was $92.2 \%$. Cardiac dysfunction developed in 4 of 78 (5.1\%) evaluable patients.

Conclusion: Access to anti-HER2 therapy in the treatment of non-metastatic HER2positive breast cancer in resource-constrained settings has improved significantly with the availability of the biosimilar trastuzumab. Imbalances in patient profiles at baseline
Correspondence to: Dr Ashish Singh Email: todrashish@gmail.com

ecancer 2021, 15:1207

https://doi.org/10.3332/ecancer.2021.1207

Published: $22 / 03 / 2021$

Received: 23/10/2020

Publication costs for this article were supported by ecancer (UK Charity number 1176307).

Copyright: (c) the authors; licensee ecancermedicalscience. This is an Open Access article distributed under the terms of the Creative Commons Attribution License (http:// creativecommons.org/licenses/by/3.0), which permits unrestricted use, distribution, and reproduction in any medium, provided the original work is properly cited. 
in routine clinical practice led to inconclusive outcomes of $\leq 12$ weeks versus $>12$ weeks trastuzumab treatment. However, on the basis of historical data, patients could be offered shorter duration of trastuzumab when a standard 1-year treatment of adjuvant trastuzumab is not feasible in resource-constrained settings. The $\mathrm{p}-\mathrm{CR}$ using the biosimilar trastuzumab in neoadjuvant treatment has been observed to be comparable to the innovator trastuzumab.

Keywords: biosimilar, breast cancer, non-metastatic, neo-adjuvant, short duration, trastuzumab

\section{Background}

The non-remunerable expenditure for cancer therapies in the developing world often highlights the glaring imbalance in the differential access to these therapies. Human epidermal growth factor receptor 2 (HER2)-positive breast cancer accounts for $26 \%-50 \%$ of all breast cancers in the Indian population and is associated with an inferior survival outcome mostly attributable to lack of access to an appropriate anti-HER2 therapy [1]. The use of trastuzumab has resulted in an improvement in disease free survival (DFS) and overall survival (OS) of patients with HER2-positive non-metastatic breast cancer which has been observed to be consistent even on a long-term follow-up [2-5]. The current standard of care is 1 year of adjuvant trastuzumab treatment [6].

Shorter durations of trastuzumab have also been evaluated in several studies [7-13]. In the FINHER study, 9 weeks of adjuvant trastuzumab improved distant DFS when compared with no trastuzumab [8]. Few randomised studies of shorter duration trastuzumab; 6 months (PHARE) and 9 weeks (Hellenic Oncology Research Group, Short-HER and SOLD) failed to prove their non-inferiority when compared to 12 months in terms of DFS [10-13]. However, the absolute differences in DFS between shorter duration and 1-year trastuzumab arms were small $(1 \%-2 \%)$ across these studies $[10,13]$. A subgroup analysis of the PHARE study suggested that the additional benefit of 1 year of trastuzumab over 6 months may be irrelevant in very low-risk subgroup [14]. Subsequently, the PERSEPHONE study demonstrated non-inferiority of 6 months versus 1-year adjuvant trastuzumab treatment [7]. Pathological complete response ( $p$-CR) is an important surrogate marker in HER2-positive breast cancer which leads to reduced recurrence rate and improved rates of DFS and OS [15, 16]. The delivery of trastuzumab in non-metastatic breast cancer in low- and middle-income countries is a challenge because of limited access, resource constraints and high cost due to a non-reimbursable, out-of-pocket expenditure pattern of healthcare provision [17, 18]. A prior analysis from India has shown that only $35.8 \%$ of eligible HER2-positive patients could receive trastuzumab as part of routine care, because of financial constraints [19]. Intent of this retrospective analysis was firstly, to identify if in resource constraint setting $\leq 12$ weeks is useful over $>12$ weeks of trastuzumab treatment. Secondly, if affordable biosimilar could be used in place of innovator to get similar outcomes in routine clinical practice.

Here, we report the pattern of use of neoadjuvant trastuzumab (innovator and biosimilar), p-CR rates and survival outcomes along with a sufficient follow-up period helpful to draw meaningful conclusions.

\section{Patients and methods}

\section{Design and study population}

We analysed the outcomes among all patients who received treatment for HER2 overexpressed breast cancer at our institution from October 2010 to August 2017. Consecutive patients diagnosed with HER2 overexpressed operable breast cancer between the years 2010 and 2017 were included in the analysis. Data was collected from the electronic medical records (EMR) and patient charts. Follow-up data was extracted from the EMR and attempts were made to contact each patient by telephone. This retrospective study was approved by the institutional review board (IRB number: 9297) on 5 February 2015.

Patients with non-metastatic T1-T4, NO-N3 and MO, HER2-positive breast cancer were included in this analysis. HER2-positivity was defined as either immunohistochemical score of 3+ and/or amplification by fluorescent in situ hybridisation using CAP 2007 criteria [20]. 


\section{Treatment}

The chemotherapy consisted of four cycles of anthracycline based chemotherapy: epirubicin $\left(90 \mathrm{mg} / \mathrm{m}^{2}\right)$ or doxorubicin $\left(60 \mathrm{mg} / \mathrm{m}^{2}\right)$ with cyclophosphamide $\left(600 \mathrm{mg} / \mathrm{m}^{2}\right)$ given in 3-weekly or dose dense 2 -weekly schedule and either 12 doses of weekly paclitaxel (80 mg/m²) or 4 doses of dose dense 2-weekly paclitaxel $\left(175 \mathrm{mg} / \mathrm{m}^{2}\right)$ with or without trastuzumab in the neoadjuvant setting in either sequence. Patients with oestrogen receptor and/or progesterone receptor positive tumours received standard adjuvant endocrine therapy (aromatase inhibitor in post-menopausal and tamoxifen in pre-menopausal patients) continued for at least 5 years. Locoregional treatment including surgery and radiotherapy was performed in all patients in this analysis.

\section{Study assessments and analysis}

The objective of our analysis was to evaluate $\mathrm{p}-\mathrm{CR}$ rates, DFS, OS and toxicity in this cohort of patients. DFS was defined as the interval between histopathological diagnosis and the first DFS event. Patients who did not experience a DFS event at the time of data cut-off or last follow-up were censored. The DFS events during follow-up included: an ipsilateral or contralateral invasive breast cancer, recurrence at any site, a second invasive cancer at any site or death due to any cause, whichever occurred first. The other endpoints were OS (time interval between histopathological diagnosis and death due to any cause), p-CR (ypTO/is, NO; defined as an absence of invasive cancer in the breast and lymph nodes in the surgical specimen) in those undergoing neoadjuvant therapy, and adverse event rate including cardiac toxicity. DFS and OS were estimated by the Kaplan-Meier method using the IBM SPSS ${ }^{\circ}$ software (version 23.0).

\section{Results}

\section{Patients and treatment}

The medical records of 135 patients who were diagnosed with operable HER2-positive breast cancer between October 2010 and August 2017 at our centre were evaluated with a data cut-off in April 2020. The median age was 51 years (range: 23-82); 57\% were postmenopausal, $31.8 \%$ were hormone receptor positive and $62.9 \%$ had stage III disease.

The baseline characteristics of the patients are shown in Table 1. Out of 135 patients, 104 (77\%) received trastuzumab (69 neoadjuvant \pm adjuvant and 35 adjuvant). Seventy (67\%) patients received biosimilar trastuzumab manufactured by Biocon Biologics, while 34 (33\%) received the innovator trastuzumab. Ninety-nine (73\%) patients received chemotherapy before surgery. Thirty-one (23\%) were treated with chemotherapy alone, as they could not receive trastuzumab due to financial constraints.

The patients were grouped for survival analysis into three groups based on the duration of use of trastuzumab (Table 1). Thirty-one (22.9\%) patients received trastuzumab for a duration shorter than 12 weeks, 73 (54.0\%) patients were treated for more than 12 weeks and 31 (23\%) did not receive any trastuzumab. The groups were not balanced with respect to their baseline disease stage with $74 \%$ of patients having stage III disease in the 'less than 12 weeks' trastuzumab cohort. The chemotherapy details are provided in Table 2.

\section{Response, disease-free survival and overall survival}

Eighty-seven patients were evaluated for $\mathrm{p}-\mathrm{CR}$, of which 69 received trastuzumab (21 - innovator trastuzumab and 48 - biosimilar trastuzumab) and 18 received only chemotherapy. The overall p-CR rate among patients who received neoadjuvant trastuzumab with chemotherapy was $37.6 \%$. Patients receiving innovator and biosimilar trastuzumab showed a p-CR of $28.5 \%$ and $41.7 \%$, respectively. Patients who only received the chemotherapy had a $\mathrm{p}-\mathrm{CR}$ rate of $22.2 \%$ (Table 3 ).

The median follow-up was 42 months (range: 3-114 months). Follow-up data was available for $78.5 \%$ patients for up to at least 2 years and for $61.4 \%$ patients for up to 3 years. There were 18 DFS events and 12 OS events. As we were unable to follow-up nearly $40 \%$ of patients for 3 years, the number of events in DFS and OS data is probably underestimated. 
The estimated (Kaplan-Meier analysis) 3-year DFS was $87.1 \%$ and the 3 -year OS was $92.2 \%$. DFS and OS for $<12$ weeks and $>12$ weeks are given in Figures 1 and 2.

Table 1. Patient characteristics.

\begin{tabular}{|c|c|c|c|}
\hline Characteristic & $\begin{array}{l}\text { No trastuzumab } \\
\qquad N=31\end{array}$ & $\begin{array}{l}\text { Short duration trastuzum- } \\
\qquad \begin{array}{c}\text { ab ( } \leq 12 \text { weeks) } \\
N=31\end{array}\end{array}$ & $\begin{array}{l}\text { Longer duration trastuzumab } \\
\qquad \begin{array}{c}\text { (>12 weeks) } \\
N=73\end{array}\end{array}$ \\
\hline Trastuzumab duration weeks (mean, range) & nil & $10(2-12)$ & $45(14-52)$ \\
\hline Age (Median) & 53 years & 50 years & 51 years \\
\hline Female, $n(\%)$ & $31(96.8 \%)$ & $31(100 \%)$ & $73(100 \%)$ \\
\hline Male, $n(\%)$ & $1(3.2 \%)$ & - & - \\
\hline \multicolumn{4}{|l|}{ Menopausal status, $\mathbf{n}(\%)$} \\
\hline Premenopausal & $11(35.5 \%)$ & $15(48.4 \%)$ & $32(43.8 \%)$ \\
\hline Postmenopausal & $20(64.5 \%)$ & $16(51.6 \%)$ & $41(56.2 \%)$ \\
\hline \multicolumn{4}{|l|}{ T stage (clinical), $n$ (\%) } \\
\hline Tx & 0 & $1(3.2 \%)$ & $1(1.4 \%)$ \\
\hline T1 & $2(6.5 \%)$ & $1(3.2 \%)$ & $5(6.8 \%)$ \\
\hline T2 & $13(41.9 \%)$ & $5(16.1 \%)$ & $30(41.1 \%)$ \\
\hline T3 & $5(16.1 \%)$ & $4(12.9 \%)$ & $10(13.7 \%)$ \\
\hline T4 & $11(35.5 \%)$ & $18(58.1 \%)$ & 27 (37.0\%) \\
\hline \multicolumn{4}{|l|}{ N stage (clinical), $n(\%)$} \\
\hline NO & $10(32.3 \%)$ & $6(19.4 \%)$ & $15(20.5 \%)$ \\
\hline N1 & 15 (48.4\%) & $13(41.9 \%)$ & 30 (41.1\%) \\
\hline N2 & $6(19.4 \%)$ & $10(32.3 \%)$ & 22 (30.1\%) \\
\hline N3 & - & - & $6(8.2 \%)$ \\
\hline \multicolumn{4}{|l|}{ AJCC stage, $n(\%)$} \\
\hline IA & $1(3.2 \%)$ & $1(3.2 \%)$ & $3(4.1 \%)$ \\
\hline IB & $1(3.2 \%)$ & - & - \\
\hline IIA & $6(19.4 \%)$ & $1(3.2 \%)$ & 12 (16.4\%) \\
\hline IIB & $7(22.6 \%)$ & $4(12.9 \%)$ & $12(16.4 \%)$ \\
\hline IIIA & $5(16.1 \%)$ & $5(16.1 \%)$ & $17(23.3 \%)$ \\
\hline IIIB & $11(35.5 \%)$ & $18(58.1 \%)$ & $23(31.5 \%)$ \\
\hline IIIC & - & - & $6(8.2 \%)$ \\
\hline \multicolumn{4}{|l|}{ Grade of tumour, $n(\%)$} \\
\hline Grade 1 & - & $2(6.5 \%)$ & $3(4.1 \%)$ \\
\hline Grade 2 & $13(41.9 \%)$ & $18(58.1 \%)$ & $33(45.2 \%)$ \\
\hline Grade 3 & 18 (58.1\%) & $11(35.5 \%)$ & $37(50.7 \%)$ \\
\hline
\end{tabular}


Table 2. Treatment details.

\begin{tabular}{|c|c|c|}
\hline Treatment characteristics & $n$ & (\%) \\
\hline \multicolumn{3}{|l|}{ Histopathology of tumour } \\
\hline Ductal carcinoma & 135 & 100 \\
\hline \multicolumn{3}{|l|}{ Anti-HER2 therapy } \\
\hline Received any trastuzumab (innovator + biosimilar) & 104 & 77 \\
\hline Biosimilar trastuzumab & 70 & 52 \\
\hline Did not receive trastuzumab & 31 & 23 \\
\hline \multicolumn{3}{|l|}{ Chemotherapy regimens } \\
\hline Anthracycline + cyclophosphamide $\rightarrow$ Paclitaxel + trastuzumab & 87 & 64.4 \\
\hline Anthracycline + cyclophosphamide $\rightarrow$ Paclitaxel & 28 & 20.7 \\
\hline Paclitaxel + trastuzumab & 8 & 5.9 \\
\hline Taxane + carboplatin + trastuzumab & 6 & 4.4 \\
\hline Anthracycline + cyclophosphamide & 3 & 2.2 \\
\hline Anthracycline + cyclophosphamide + trastuzumab & 2 & 1.5 \\
\hline Trastuzumab + letrozole & 1 & 0.7 \\
\hline \multicolumn{3}{|l|}{ Chemotherapy dosing frequency $(N=40)$} \\
\hline Dose-dense (every 2 weeks) & 60 & 44.4 \\
\hline Every 3 weeks & 75 & 55.6 \\
\hline \multicolumn{3}{|l|}{ Growth factor support } \\
\hline Peglylated GCSF given & 113 & 83.7 \\
\hline No GCSF support & 22 & 16.3 \\
\hline
\end{tabular}

Table 3. Pathological assessment post neoadjuvant treatment.

\begin{tabular}{|l|c|c|}
\hline \multicolumn{1}{|c|}{ Pathological response } & $n(N)$ & \\
\hline$p$-CR (ypT0/is N0) & & \\
\hline$p$-CR with neoadjuvant chemotherapy and trastuzumab & $26(69)$ & 37.6 \\
\hline$p$-CR with neoadjuvant chemotherapy and innovator trastuzumab & $6(21)$ & 28.5 \\
\hline$p$-CR with neoadjuvant chemotherapy and biosimilar trastuzumab & $20(48)$ & 41.7 \\
\hline$p$-CR with neoadjuvant chemotherapy without trastuzumab & $4(18)$ & 22.2 \\
\hline p-CR overall & $30(87)$ & 34.5 \\
\hline Breast p-CR (ypT0/is N0/+) & 42 & 40.3 \\
\hline Nodal p-CR (ypN0) & 60 & 57.7 \\
\hline N, Total number of patients evaluated for p-CR; $n$, Number of patients achieving p-CR & \\
\hline Miller Payne & 86 & \\
\hline Miller Payne grade 1 & 3 & 3.5 \\
\hline Miller Payne grade 2 & 8 & 9.3 \\
\hline Miller Payne grade 3 & 7 & 8.1 \\
\hline Miller Payne grade 4 & 26 & 30.2 \\
\hline Miller Payne grade 5 & 42 & 48.8 \\
\hline
\end{tabular}




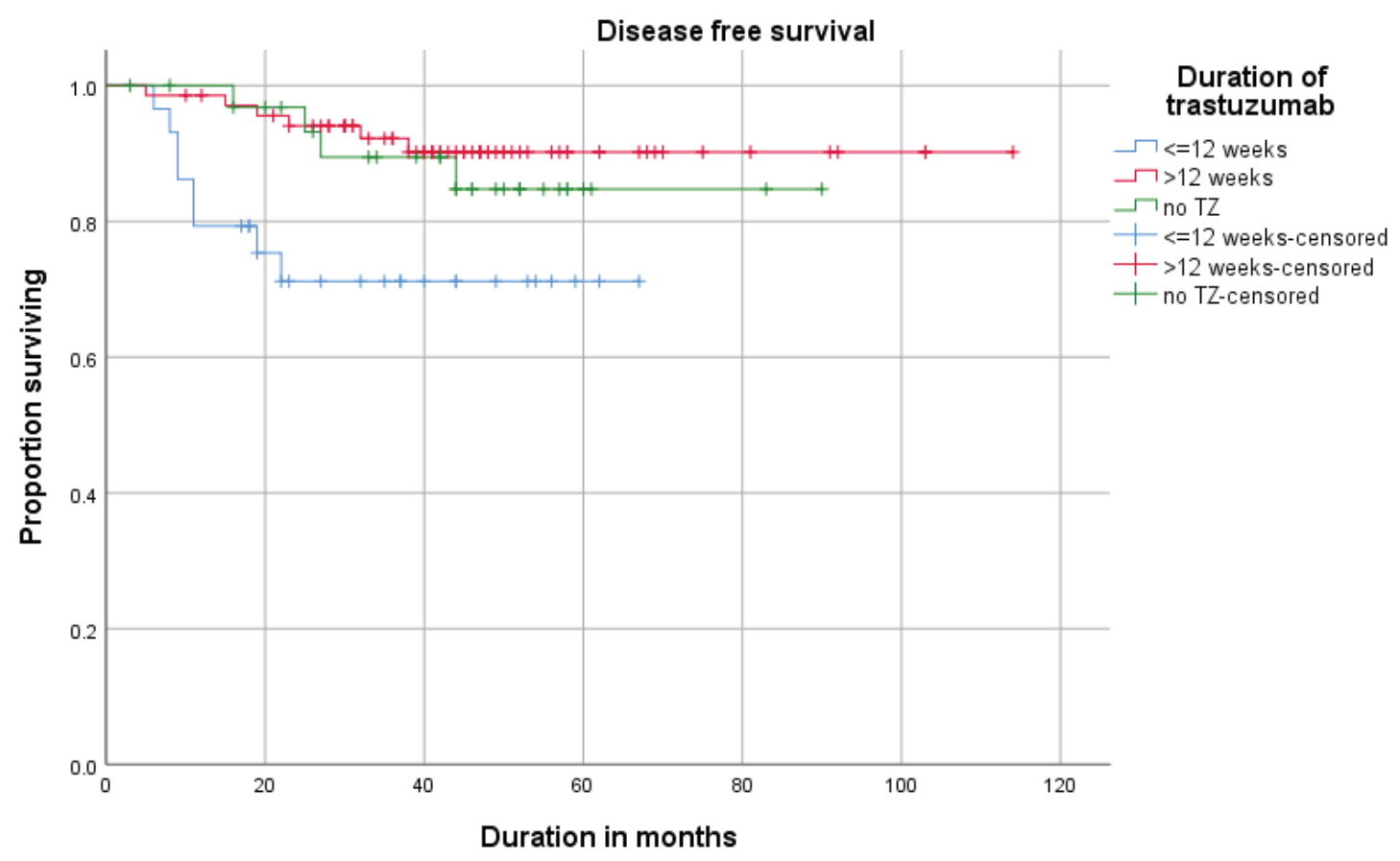

Figure 1. Disease free survival (DFS) of patients receiving neoadjuvant \pm adjuvant or adjuvant trastuzumab ( $\leq 12$ weeks vs $>12$ weeks).

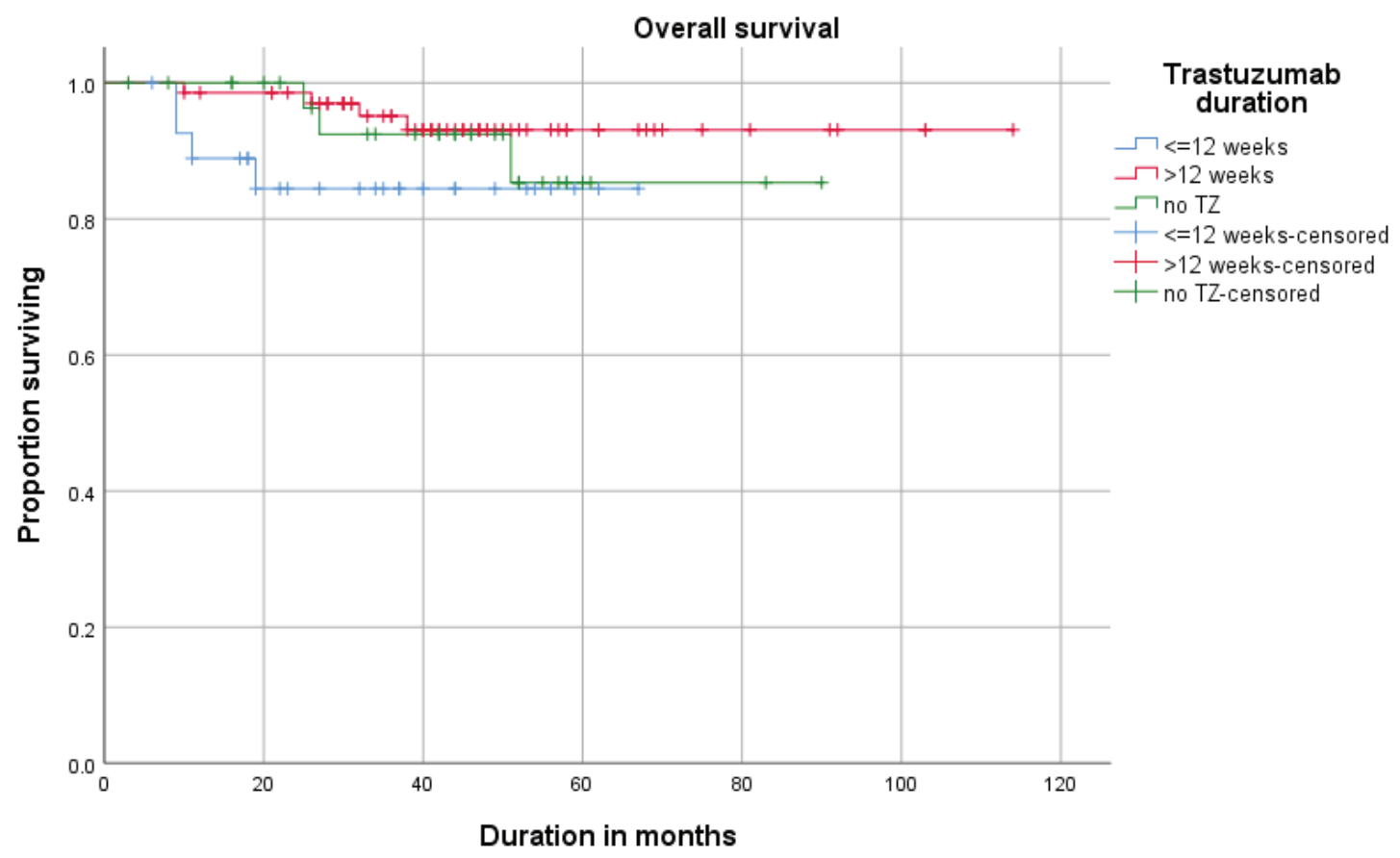

Figure 2. Overall survival (OS) of patients receiving neo adjuvant \pm adjuvant or adjuvant trastuzumab ( $\leq 12$ weeks vs $>12$ weeks). 


\section{Adverse events}

The toxicity data was collected from the medical records. Grade III/IV peripheral neuropathy was seen in four patients among whom neuropathy was documented. A cardiac event was defined as a reduction in left ventricular ejection fraction of $\geq 10 \%$ from baseline. At the time of analysis, 4 of 78 (5.1\%) evaluable patients had experienced an asymptomatic decline in the ejection fraction. No patient had symptomatic cardiac dysfunction and no patient died due to cardiotoxicity. Long-term follow-up data for cardiac dysfunction was not available.

\section{Discussion}

Biosimilar trastuzumab has been approved for use in advanced breast cancer and has significantly improved access to anti-HER2 therapy in India. This is the first report of its efficacy in a neoadjuvant setting in non-metastatic breast cancer.

Neoadjuvant trastuzumab with chemotherapy improves p-CR, reduces the risk of relapse, disease progression and death. Gianni et al [16] in the NOAH study have reported a p-CR rate of $43 \%$ versus $22 \%$ with trastuzumab in neoadjuvant setting compared to chemotherapy alone, respectively, in patients with newly diagnosed locally advanced breast cancer. In another study, Minckwitz et al [21] reported that sequential anthracycline-taxane-based chemotherapy in combination with trastuzumab resulted in a p-CR of $40 \%$ compared to a p-CR of $17 \%$ with the chemotherapy alone. In this real-world scenario, patients receiving neoadjuvant chemotherapy with any of the two trastuzumab's showed an overall $\mathrm{p}-\mathrm{CR}$ rate of $37.6 \%$, with innovator and biosimilar trastuzumab resulting in a p-CR of $28.5 \%$ and $41.7 \%$, respectively. Our cohort of patients had higher $\mathrm{p}-\mathrm{CR}$ rates, irrespective of the number of cycles of neoadjuvant trastuzumab provided, as compared to prior smaller cohorts reported previously from India (34.2\% and 36.3\%) [22, 23]. While, OS and progression-free survival (PFS) could not be calculated for biosimilar versus innovator because of missing data and low sample size. However, as per recent meta-analysis published in 2020 , achieving p-CR following neoadjuvant chemotherapy (NACT) is correlated with improved event free survival and OS particularly in HER2 +ve and triple negative breast cancer [24].

The present report, which includes 64\% locally advanced breast cancer patients, shows a 3-year DFS of $87.1 \%$ and an OS of $92.2 \%$, using an anthracycline and taxane based chemotherapy in combination with a shorter duration treatment with trastuzumab. This is in contrast with the DFS and OS outcomes after 1 year of trastuzumab treatment as reported in the HERceptin Adjuvant study and the combined NSABP B31 and NCCTG N9831 results at 4 years, ranging from 79\%\%-85\% and 89\%\%-91\%, respectively [3, 25].

Our cohort of women included a higher proportion of patients with advanced breast cancer as compared to the prior reported trials, which had a larger proportion of early breast cancers. Our follow-up duration, however, is shorter at 42 months and only two-thirds of our patients could be followed-up for 3 years. This is due to the fact that the patients preferred to report at a local hospital closer to their homes for a follow-up.

Since majority of these women presented with locally advanced breast cancer, it is imperative to compare our results with the pertinent subgroup analyses from randomised trials using adjuvant trastuzumab. In the latest joint analysis report of the NCCTG N9831 and NSABP B-31 trials, the 4-year DFS of patients with tumour size greater than $5 \mathrm{~cm}$ was $52 \%$ in the control group and $78 \%$ with 1 year of trastuzumab treatment [5]. In a smaller phase II randomised study (E2198) comparing 12 weeks of trastuzumab treatment with 1 year treatment in patients with stage II and IIIA disease, the 5-year DFS was $74 \%$ and $78 \%$ and OS was $91 \%$ and $89 \%$, respectively. This study did not find a significant difference in the two groups, though it was inadequately powered for significance [26]. Recent retrospective study in Ontario reported that less than 1 year of trastuzumab treatment increases risk of relapse and death [27]. The phase III randomised trials with shorter duration of trastuzumab treatment (ranging from 9 weeks to 6 months), including the more recent PERSEPHONE trial, report non-inferiority to 1-year trastuzumab treatment $[7,8,10,11,13]$. Although the optimal duration of adjuvant trastuzumab is still debatable, 1 year remains the standard of care as of today. The results of our study suggest that a regimen with a shorter duration of trastuzumab treatment improves outcomes compared to historical controls of patients not receiving trastuzumab. In the real-world setting of low middle-income groups, inclusion of biosimilar trastuzumab, albeit for a shorter duration, in advanced non-metastatic patients can lead to improved p-CR and OS. 
In the clinical trials using shorter duration of trastuzumab (6 months and 9 weeks), the incidence of cardiac dysfunction was $3.4 \%$ and $5 \%$ and that of congestive heart failure around $0.5 \%$; in contrast to $4 \%$ in the NSABP31 study where the treatment was of 1 year duration [10, 13 , 28 . Retrospective analyses among patient cohorts in routine clinical practice describe the incidence of symptomatic heart failure at $3 \%$ and $6.6 \%$ among patients receiving sequential therapy [29,30]. Also, in this real-world cohort, the incidence of a major cardiac event was higher (22.1\% versus $17 \%$ versus $6.5 \%$ ) among patients receiving sequential taxanes/trastuzumab followed by anthracycline versus those receiving trastuzumab without anthracyclines versus those receiving anthracyclines without trastuzumab [30]. The incidence of cardiac toxicity in our patient cohort receiving shorter duration of trastuzumab was as expected at $5.1 \%$ (4 patients). The overall safety profile was similar in patients who received trastuzumab. The higher incidence of cardiac failure in our analysis could be attributable to higher incidence of comorbid conditions including cardiac predisposition in a real-world population compared to those observed in clinical trials. This may also be attributable to a higher proportion of patients receiving sequential anthracycline and trastuzumab (64.4\%). Though other real-world cohorts describe delayed cardiac events related to trastuzumab, our lack of routine long-term cardiac follow-up did not reflect this trend with all four major cardiac events occurring less than 10 months from the end of adjuvant chemotherapy. Even though this is a retrospective real-world analysis with many possible confounders, the optimal sequencing of anthracyclines and taxanes-trastuzumab with respect to cardiac toxicity needs to be further evaluated, especially in the setting of shorter duration trastuzumab.

As with other real-world datasets, this analysis has limitations of its retrospective nature, small sample size, short follow-up duration and missing data points. In patients with locally advanced disease and limited access to trastuzumab, all attempts were made to provide them at least 12 weeks of trastuzumab, which is evident in the selection bias with more advanced disease receiving shorter duration trastuzumab. The relative efficacy and cost effectiveness of 12 weeks of trastuzumab versus no trastuzumab or 1 year of trastuzumab cannot be discerned from this analysis because of base line imbalances with $74 \%$ patients having stage III disease in $\leq 12$ weeks cohort. However, compared with the historical controls of patients who have not received any trastuzumab, the use of short duration trastuzumab ( $\leq 12$ weeks) regimen can be considered as a viable option in resource-constrained settings to improve $\mathrm{p}$-CR and OS. While 1-year trastuzumab remains the standard of care for non-metastatic HER2-positive breast cancer, shorter duration of trastuzumab treatment warrants further evaluation. In this analysis, only one biosimilar (Biocon Biologics Biosimilar) was compared against innovator, which was non-inferior for $\mathrm{p}$-CR outcomes. During the study period, only Biocon Biologics biosimilar was available in the formulary, hence only this biosimilar was used to compare with the innovator. The reason of comparison of innovator versus biosimilar was socioeconomic so that all patients can afford the complete treatment. The biosimilar brand, which was available in the formulary, was the closest to the innovator in terms of safety, efficacy, purity and potency. This biosimilar was approved by the United States Food and Drug Administration (USFDA) and proved equivalence with the innovator in a global large multi-centric randomised trial. In India, over 75\% cancer expenses are non-remunerable and out of pocket [31]. Additionally, trastuzumab is not funded by any insurance schemes [32]. The availability of a safe and cost effective biosimilar trastuzumab with proven quality can help more and more patients with advanced disease to access this essential treatment.

\section{Conclusion}

Early introduction of trastuzumab in the neoadjuvant setting in HER2-positive non-metastatic breast cancer improves p-CR. Our results suggest that when the standard 1 year of adjuvant trastuzumab is not feasible, a regimen that incorporates shorter duration of trastuzumab (including biosimilar) delivered concurrently with taxanes and sequentially with anthracyclines is a reasonable alternative. Access to anti HER2 therapy in the treatment of non-metastatic HER2 overexpressed breast cancer in resource-constrained settings has improved significantly with the availability of the biosimilar trastuzumab. This study reports comparable p-CR with the biosimilar trastuzumab versus the innovator. An improvement in DFS and OS in all patients who received trastuzumab was similar to historical data.

While this is the first report of the biosimilar trastuzumab in non-metastatic breast cancer in the neoadjuvant setting, it carries a limitation of being a non-randomised study with retrospective data. 


\section{Conflict of interests}

The author(s) declare that they have no conflict of interest.

\section{Funding}

This research received no external funding.

\section{References}

1. Bahl A, Singh R, and Wadhwa J, et al (2018) Practical consensus recommendations regarding the management of HER2 neu positive early breast cancer South Asian J Cancer 7(2) 146-150 https://doi.org/10.4103/sajc.sajc_123_18

2. Piccart-Gebhart MJ, Procter M, and Leyland-Jones B, et al (2005) Trastuzumab after adjuvant chemotherapy in HER2-positive breast cancer N Engl J Med 353(16) 1659-1672 https://doi.org/10.1056/NEJMoa052306 PMID: 16236737

3. Romond EH, Perez EA, and Bryant J, et al (2005) Trastuzumab plus adjuvant chemotherapy for operable HER2-positive breast cancer N Engl J Med 353(16) 1673-1684 https://doi.org/10.1056/NEJMoa052122 PMID: 16236738

4. Cameron D, Piccart-Gebhart MJ, and Gelber RD, et al (2017) 11 years' follow-up of trastuzumab after adjuvant chemotherapy in HER2-positive early breast cancer: final analysis of the HERceptin adjuvant (HERA) trial Lancet 389(10075) 1195-1205 https://doi. org/10.1016/S0140-6736(16)32616-2 PMID: 28215665 PMCID: 5465633

5. Perez EA, Romond EH, and Suman VJ, et al (2011) Four-year follow-up of trastuzumab plus adjuvant chemotherapy for operable human epidermal growth factor receptor 2-positive breast cancer: joint analysis of data from NCCTG N9831 and NSABP B-31 J Clin Oncol 29(25) 3366-3373 https://doi.org/10.1200/JCO.2011.35.0868 PMID: 21768458 PMCID: 3164242

6. Denduluri N, Somerfield MR, and Eisen A, et al (2016) Selection of optimal adjuvant chemotherapy regimens for human epidermal growth factor receptor 2 (HER2) -negative and adjuvant targeted therapy for HER2-positive breast cancers: an American society of clinical oncology guideline adaptation of the cancer care Ontario clinical practice guideline J Clin Oncol 34(20) 2416-2427 https://doi. org/10.1200/JCO.2016.67.0182 PMID: 27091714

7. Earl HM, Hiller L, and Vallier A-L, et al (2018) PERSEPHONE: 6 versus 12 months (m) of adjuvant trastuzumab in patients (pts) with HER2 positive (+) early breast cancer (EBC): randomised phase 3 non-inferiority trial with definitive 4-year (yr) disease-free survival (DFS) results J Clin Oncol 36(15) 506 https://doi.org/10.1200/JCO.2018.36.15_suppl.506

8. Joensuu H, Kellokumpu-Lehtinen PL, and Bono P, et al (2006) Adjuvant docetaxel or vinorelbine with or without trastuzumab for breast cancer N Engl J Med 354(8) 809-820 https://doi.org/10.1056/NEJMoa053028 PMID: 16495393

9. Joensuu H, Bono P, and Kataja V, et al (2009) Fluorouracil, epirubicin, and cyclophosphamide with either docetaxel or vinorelbine, with or without trastuzumab, as adjuvant treatments of breast cancer: final results of the FinHer trial J Clin Oncol 27(34) 5685-5692 https:// doi.org/10.1200/JCO.2008.21.4577 PMID: 19884557

10. Pivot X, Romieu G, and Debled M, et al (2013) 6 months versus 12 months of adjuvant trastuzumab for patients with HER2-positive early breast cancer (PHARE): a randomised phase 3 trial Lancet Oncol 14(8) 741-748 https://doi.org/10.1016/S1470-2045(13)70225-0 PMID: 23764181

11. Mavroudis D, Saloustros E, and Malamos N, et al (2015) Six versus 12 months of adjuvant trastuzumab in combination with dose-dense chemotherapy for women with HER2-positive breast cancer: a multicenter randomized study by the Hellenic Oncology Research Group (HORG) Ann Oncol 26(7) 1333-1340 https://doi.org/10.1093/annonc/mdv213 PMID: 25935793 
12. Joensuu H, Fraser J, and Wildiers H, et al (2018) Abstract GS3-04: a randomized phase III study of adjuvant trastuzumab for a duration of 9 weeks versus 1 year, combined with adjuvant taxane-anthracycline chemotherapy, for early HER2-positive breast cancer (the SOLD study). Cancer Res 78(4)

13. Conte P, Frassoldati A, and Bisagni G, et al (2018) Nine weeks versus 1 year adjuvant trastuzumab in combination with chemotherapy: final results of the phase III randomized Short-HER study Ann Oncol 29(12) 2328-2333 https://doi.org/10.1093/annonc/mdy414 PMID: 30219886

14. Kramar A, Bachelot T, and Madrange N, et al (2014) Trastuzumab duration effects within patient prognostic subgroups in the PHARE trial Ann Oncol 25(8) 1563-1570 https://doi.org/10.1093/annonc/mdu177 PMID: 24827132

15. Untch M, Fasching AP, and Konecny EG, et al (2011) Pathologic complete response after neoadjuvant chemotherapy plus trastuzumab predicts favorable survival in human epidermal growth factor receptor 2-overexpressing breast cancer: results from the TECHNO trial of the AGO and GBG study groups J Clin Oncol 29(25) 3351-3357 https://doi.org/10.1200/JCO.2010.31.4930 PMID: 21788566

16. Gianni L, Eiermann W, and Semiglazov V, et al (2010) Neoadjuvant chemotherapy with trastuzumab followed by adjuvant trastuzumab versus neoadjuvant chemotherapy alone, in patients with HER2-positive locally advanced breast cancer (the NOAH trial): a randomised controlled superiority trial with a parallel HER2-negative cohort Lancet 375(9712) 377-384 https://doi.org/10.1016/ S0140-6736(09)61964-4 PMID: 20113825

17. Ghosh J, Gupta S, and Desai S, et al (2011) Estrogen, progesterone and HER2 receptor expression in breast tumors of patients, and their usage of HER2-targeted therapy, in a tertiary care centre in India Indian J Cancer 48(4) 391-396 https://doi.org/10.4103/0019$\underline{509 X .92245}$

18. Reeder-Hayes K, Hinton SP, and Meng K, et al (2016) Disparities in use of human epidermal growth hormone receptor 2-targeted therapy for early-stage breast cancer J Clin Oncol 34(17) 2003-2009 https://doi.org/10.1200/JCO.2015.65.8716 PMID: 27069085 PMCID: $\underline{4966516}$

19. Adusumilli P, Konatam ML, and Gundeti S, et al (2017) Treatment challenges and survival analysis of human epidermal growth factor receptor 2-positive breast cancer in real world Indian J Med Paediatr Oncol 38(1) 22-27 https://doi.org/10.4103/0971-5851.203511 PMID: 28469333 PMCID: 5398102

20. Wolff AC, Hammond MEH, and Hicks DG, et al (2013) Recommendations for human epidermal growth factor receptor 2 testing in breast J Clin Oncol 31(31) 3997-4013 https://doi.org/10.1200/JCO.2013.50.9984 PMID: 24101045

21. Von Minckwitz G, Loibl S, and Untch M (2012) What is the current standard of care for antiHER2 neoadjuvant therapy in breast cancer? Oncology 26(1) 20-26 PMID: 22393792

22. Tiwari A, Gogia A, and Deo S, et al (2017) Retrospective study of efficacy and safety of neoadjuvant docetaxel, carboplatin, and trastuzumab in HER2-positive locally advanced and oligometastatic breast cancer: an Indian experience Indian J Cancer 54(1) 343 https://doi. org/10.4103/ijc.IJC_152_17 PMID: 29199719

23. Iyer $P$, Radhakrishnan $V$ and Balasubramanian $A$, et al (2020) Study of pathological complete response rate with neoadjuvant concurrent chemoradiation with paclitaxel in locally advanced breast cancer Indian J Cancer 57(4) 428-434 PMID: 32675440

24. Spring LM, Fell G, and Arfe A, et al (2020) Pathologic complete response after neoadjuvant chemotherapy and impact on breast cancer recurrence and survival: a comprehensive meta-analysis Clin Cancer Res 26(12) 2838-2848 https://doi.org/10.1158/1078-0432.CCR19-3492 PMID: 32046998 PMCID: 7299787

25. Gianni L, Dafni U, and Gelber RD, et al (2011) Treatment with trastuzumab for 1 year after adjuvant chemotherapy in patients with HER2positive early breast cancer: a 4-year follow-up of a randomised controlled trial Lancet Oncol 12(3) 236-244 https://doi.org/10.1016/ S1470-2045(11)70033-X PMID: 21354370 
26. Schneider BP, O'Neill A, and Shen F, et al (2015) Pilot trial of paclitaxel-trastuzumab adjuvant therapy for early stage breast cancer: a trial of the ECOG-ACRIN cancer research group (E2198) Br J Cancer 113(12) 1651-1657 https://doi.org/10.1038/bjc.2015.405 PMID: 26625004 PMCID: 4701997

27. Rushton M, Lima I, and Tuna M, et al (2020) Impact of stopping trastuzumab in early breast cancer: a population-based study in Ontario, Canada J NatI Cancer Inst 112(12) 1222-1230 https://doi.org/10.1093/jnci/djaa054 PMID: 32343801 PMCID: 7735777

28. Tan-Chiu E, Yothers G, and Romond E et al (2005) Assessment of cardiac dysfunction in a randomized trial comparing doxorubicin and cyclophosphamide followed by paclitaxel, with or without trastuzumab as adjuvant therapy in node-positive, human epidermal growth factor receptor 2-overexpressing breast cancer: NSABP B-31 J Clin Oncol 23(31) 7811-7819 https://doi.org/10.1200/ JCO.2005.02.4091 PMID: 16258083

29. Tarantini L, Cioffi G, and Gori S, et al (2012) Trastuzumab adjuvant chemotherapy and cardiotoxicity in real-world women with breast cancer J Card Fail 18(2) 113-119 https://doi.org/10.1016/j.cardfail.2011.10.015 PMID: 22300778

30. Thavendiranathan P, Abdel-Qadir H, and Fischer HD, et al (2016) Breast cancer therapy-related cardiac dysfunction in adult women treated in routine clinical practice: a population-based cohort study J Clin Oncol 34(19) 2239-2246 https://doi.org/10.1200/ JCO.2015.65.1505 PMID: 27091709

31. Pramesh CS, Badwe RA, and Borthakur BB, et al (2014) Delivery of affordable and equitable cancer care in India Lancet Oncol 15(6) e223-e233 https://doi.org/10.1016/S1470-2045(14)70117-2 PMID: 24731888

32. Ghosh J, Gupta S, and Desai S, et al (2011) Estrogen, progesterone and HER2 receptor expression in breast tumors of patients, and their usage of HER2-targeted therapy, in a tertiary care centre in India Indian J Cancer 48(4) 391-396 https://doi.org/10.4103/0019$\underline{509 X .92245}$ 